\title{
Carbon Nanotube Functionalization Decreases Osteogenic Differentiation in Aluminum Oxide Reinforced Ultrahigh Molecular Weight Polyethylene
}

Anup Kumar Patel, Pramanshu Trivedi and Kantesh Balani ${ }^{*}$

\section{Supporting Information}

Supplementary 1. Schematic of CNT functionalization with APTES and interactions

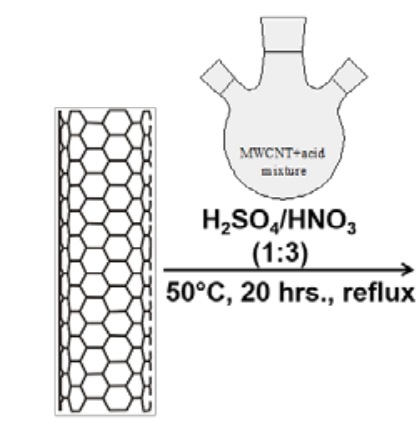

Pristine CNT

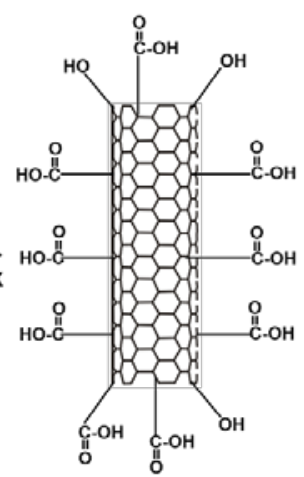

Acid treated CNT
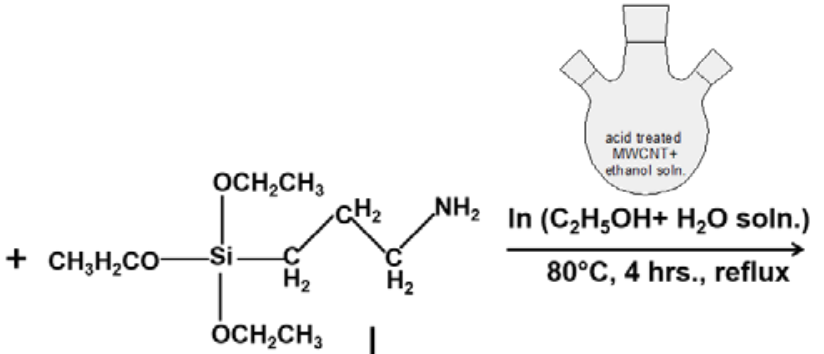

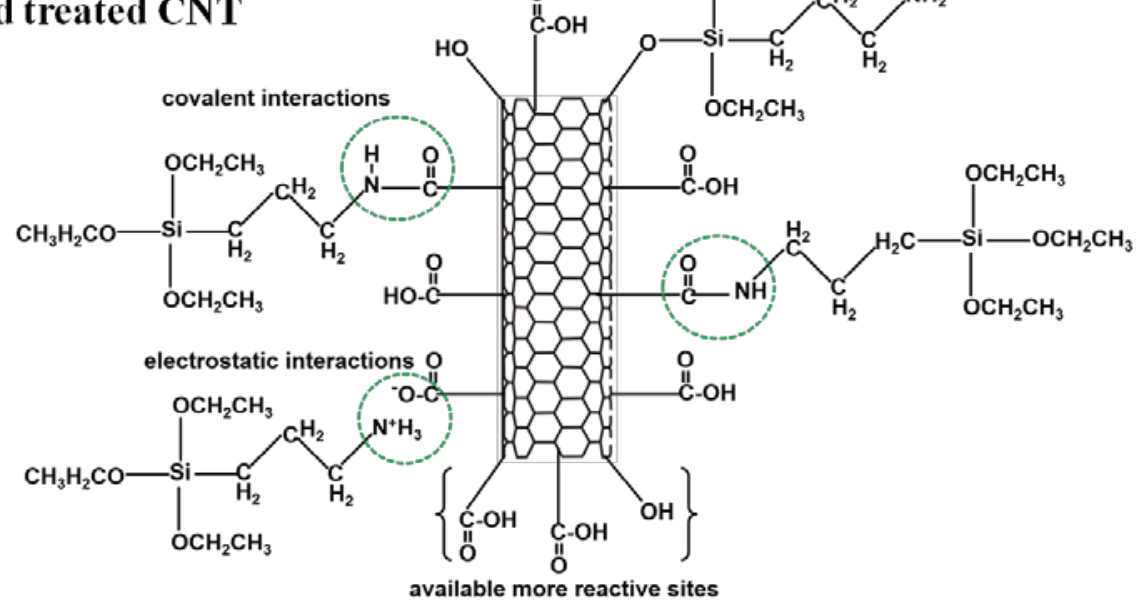

APTES functionalized CNT

*Corresponding Author. Kantesh Balani, Email: kbalani@iitk.ac.in, Ph: +91-512-259-6194 
Supplementary 2. Owen-Wendt-Rabel-Kaelble (OWRK) geometric mean equations

$$
\begin{aligned}
& \sigma_{s}=\sigma_{s 1}+\sigma_{1} \cdot \cos \theta \\
& \sigma_{1}=\sigma_{1}^{d}+\sigma_{1}^{p} \\
& \sigma_{s}=\sigma_{s}^{d}+\sigma_{s}^{p} \\
& \sigma_{s 1}=\sigma_{s}+\sigma_{1}-2\left(\sqrt{\sigma_{s}^{d} \cdot \sigma_{1}^{d}}+\sqrt{\sigma_{s}^{p} \cdot \sigma_{1}^{p}}\right) \\
& \frac{1+\cos \theta}{2} \cdot \frac{\sigma_{1}}{\sqrt{\sigma_{1}^{d}}}=\sqrt{\sigma_{s}^{p}} \sqrt{\frac{\sigma_{1}^{p}}{\sigma_{1}^{d}}}+\sqrt{\sigma_{s}^{d}}
\end{aligned}
$$

Where, $\theta$ is the contact angle between solid and liquid, $\sigma_{\mathrm{s} 1}$ is the interfacial tension between liquid and solid, $\sigma 1 \& \sigma_{\mathrm{s}}$ are the surface tension of liquids and surface energy of solid surface, $\sigma_{\mathrm{s}}^{\mathrm{p}}$ \& $\sigma_{\mathrm{s}}{ }^{\mathrm{d}}$ are the polar and dispersion components of solid respectively. 